\title{
Genetic Marker
}

National Human Genome Research Institute (NHGRI)

\section{Source}

National Human Genome Research Institute (NHGRI). Genetic Marker.

A genetic marker is a DNA sequence with a known physical location on a chromosome. Genetic markers can help link an inherited disease with the responsible gene. DNA segments close to each other on a chromosome tend to be inherited together. Genetic markers are used to track the inheritance of a nearby gene that has not yet been identified, but whose approximate location is known. The genetic marker itself may be a part of a gene or may have no known function. 